\title{
ESTABLISHING TECHNICAL AND TACTICAL PERFORMANCE GOALS FOR ELITE MALE VOLLEYBALL PLAYERS
}

\author{
José M. Palao, 1, A, C, D Antonio García-de-Alcaraz, 2, B Elena Hernández-Hernández,, , A, B \\ David Valadés, ${ }^{4, A, C}$ Enrique Ortega-Toro $5, A, C$
}

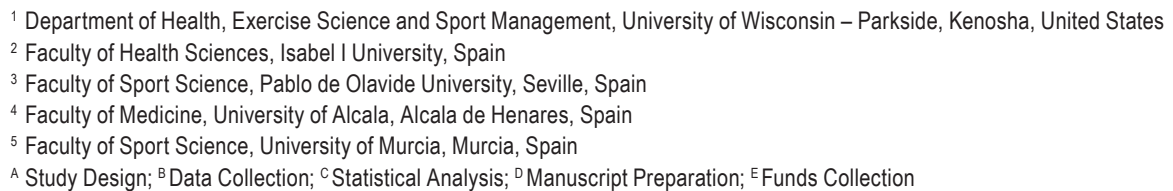

Address for correspondence:

José M. Palao

University of Wisconsin - Parkside. SAC D140A. Kenosha, WI 53,144, United States

E-mail: palaojm@gmail.com

\begin{abstract}
Ahstract The goal of this study was to establish technical and tactical performance goals for elite male volleyball players (Spanish first division). This paper is the result of scientific collaboration between volleyball coaches and researchers. The process of establishing the goals and the reference values of the performance goals was: 1) conceptual analysis, establishing the criteria for the analysis of players' and teams' performance (researchers and coaches); 2) mathematical analysis, data collection and data analysis of the possible performance goals; and 3) practical analysis, establishing the performance goals according to their usefulness and applicability following the SMART principle. Seven performance goals were established to evaluate the technical-tactical actions of the players in training and in competition. Protocols utilized can be used as references of how to calculate the technical and tactical performance goals in sport.
\end{abstract}

Key Words sport, performance, monitoring, statistics

\section{Introduction}

Match analysis is common in most field, bat, and net sports. Skill analysis can have different purposes (Coleman, 2002): to inform the media and the public, for team evaluation, team management or during matches, for match evaluation, for training evaluation, and to obtain reference values and/or patterns of the sport. Federations and competition organizers carry out match analysis to inform the media, the public, and the teams. Coaches carry out match analysis to prepare matches (e.g. to scout) and training sessions, to make decisions during matches, and to analyze their teams or other teams in competition. Researchers carry out match analysis to increase the understanding of the sport (e.g. win-lose) and to establish reference values or patterns. Currently, there is a lack 
of connection between researchers and coaches (Williams, Kendall, 2007). There are many reasons for this (e.g. channels of communication, way of communicating, interest in communicating, etc.).

One reason for this gap could be the way in which the skill analysis is done. Researchers usually analyze the data with the goal of establishing general tendencies and trends (i.e. generalization), and coaches usually analyze the data by match to prepare their team for a specific match or opponent. Both perspectives of analysis have the risk of being incomplete. Utilizing a global or general perspective risks having data compensate each other and therefore there is a loss of important details, and specific detailed analysis can result in not seeing the big picture due to certain tendencies. The two types of analysis require different protocols, instruments, etc. To improve sport performance, it is necessary to establish links between researchers and coaches. This symbiotic process can help both groups better achieve their objectives. It allows coaches to increase the power of their analysis as well as the resources involved through collaboration. Researchers are able to get familiar with the real problems found in performance sport, the terms, priorities, and ways of communication, and they get more familiar with aspects that affect performance sport.

Volleyball is not immune to this lack of connection. Reference values to guide and interpret teams' and players' actions in a match are not common (e.g. Coleman, 2002). To be useful, an evaluation requires reference values to know whether the result is adequate or not. These values are common in areas such as physical testing or conditioning. For example, in strength training, if weight and speed execution are not monitored, it is not possible to establish whether athletes are working properly (Samozino, Rejec, Prampero, Belli, Morin, 2012). Ideally, if players' skills are going to be evaluated in training and competition, reference values should be available for all levels of competition for both genders (Coleman, 2002). This lack of objective information has caused coaches to use subjective information combined with their accumulative analysis and experience to analyze players' and teams' actions in practice and in competition with regard to technique and tactics. Establishing reference values involves the generalization of the trends and characteristics of a competition. These values will be used by coaches to analyze their players and teams. In theory, the collaboration of researchers and coaches will allow them to realize a strong conceptual analysis of the performance indicators of the sport, to conduct a mathematical analysis of the performance indicators, and to establish usefulness and applicability of performance goals. This process provides an integrated approach that should provide an evidence-based applicable outcome. This research is the result of scientific support provided to volleyball coaches and this paper describes the steps taken in this collaboration process. The goal of this study was to establish technical and tactical performance goals for elite male volleyball players.

\section{Method}

Three steps in the process of calculation and analysis were done in order to establish the reference values of the goals: 1) establishing the criteria for calculating and analyzing the performance (researchers and coaches); 2) data collection (match observation) and descriptive (mean, standard deviation, baseline of won and lost sets, and percentiles) and inferential analyses (Chi-Square Test and likelihood ratio) of the data comparing won/lost sets; and 3) review of the data by coaches and researchers to establish performance goals following the SMART principle (Bull, Albinson, Shambrook, 1996).

The first stage involved the work sessions between researchers and coaches. The goal was to establish the criteria for calculating and analyzing the performance. Three coaches participated in this stage. They had more than 
five years of coaching experience in volleyball, they had the highest volleyball coaching certification Spain, and they had bachelor degrees in Sport Science. Likewise, three researchers participated in this stage. Two had more than seven years of experience in coaching volleyball, and all three were university professors and had their doctorate in Sport Science. A total of three meetings were held. The steps followed to establish performance goals from technical-tactical actions from match analysis were (Palao, López-Martínez, 2012): 1) establish the critical aspects of the sport at this level of competition; 2) establish the performance indicators and reference values for the sport; 3) establish the baseline performance indicators and characteristics of the players and teams; and 4) establish the training and competition goals. In the first meeting, there was an introductory part, a brainstorming session, and an organizing session for the tasks to do for the different parts (prepare a list of actions to analyze, criteria, and aspects that would be interesting to analyze). In the rest of the meetings, the proposed list was reviewed by coaches and researchers. At the end of the process, the actions, criteria, and manner of analysis were established. The criteria followed to establish the reference values was the consensus; at least five out of the six participants had to agree on the criteria.

The second stage involved the data collection (i.e. match observation) and data analysis. Three levels in the competition were established according to their final ranking: level 1, 1st-4th; level 2, 5th-8th, and level 3, 9th-12th. One team from each of the different levels of the competition was selected and included in the sample. All matches that the three teams played in the regular season of Spain's men's first division competition were analyzed (20082009 season). A sample of ninety sets from 44 matches was analyzed. The variables studied were the efficacy of the different actions (serve, reception, set, attack, block, and dig), the complex or game phase (side-out or defense), and the result of the set. The efficacy was measured in relation to the action's success and the options that the action gave to the attack of the analyzed team and the opponent (Coleman, Neville, Gordon, 1969). An adaptation of the FIVB statistical system and Data project system was used (Data Project, 2008). Terminal actions (serve, spike, and block) were evaluated on a 5-point scale (i.e. error, all opponent attack options, limited opponent attack options, no opponent attack options, and point). Continuity actions were evaluated on a 4-point scale (i.e. error, no attack options, limited attack options, and all attack options).

Data recording and registration was carried out using video cameras and the Data project software. Three trained observers registered the data. Observers were part of the coaching staff of professional volleyball teams with more than four years of experience in peak performance and with formal training in the use of the software. Observations of the matches were done by the observers as part of their jobs with the teams. One observer did inter- and intra-reliability testing with one of the researchers. The values found were greater than 0.82 and 0.98 , respectively, utilizing Cohen's kappa. All observers observed a match for quality control. All matches were reviewed by the referent observer to correct the differences in the criteria observed in the match trial.

Data were exported from the Data Project Software to a spreadsheet (Garcia-de-Alcaraz, Palao, 2013). From the raw data, statistical values proposed in stage one were calculated. With this data matrix, descriptive (mean, standard deviation, baseline of won and lost sets, and percentiles) and inferential (Chi-Square Test and likelihood ratio) analyses of the data to compare won/lost sets were done with the SPSS 21 software. Statistical significance was set at 0.05 .

The third stage involved a comparison of the results obtained with data from previous studies in the literature (win/loss studies and team levels studies). A review of the studies (found in Web of Knowledge, Sportdiscus, Sponet, and Google Scholar) that analyze the actions studied and the differences between winning and losing was 
done with the data found in stage two. The review and establishment of the reference values was done by the same coaches and researchers as in phase one. Coaches and researchers established performance goals following the SMART principle (Specific, Measurable, Achievable, Realistic, Trackable) (Bull et al., 1996). The criteria followed to establish the reference values was consensus, taking into account the usefulness, importance, and possibilities to implement; at least five of the six participants had to agree on the criteria.

\section{Results}

The result of the first stages of the actions, criteria, and manners of analysis is shown in Table 1. The list was composed of six skills analyzed in two phases (team in side-out and team on defense) and in the six rotations. The efficacy was measured in relation to the action's success and the options that the action gave the analyzed team and the opponent. An adaptation of the FIVB statistical system and Data project system was used to measure the efficacy (Coleman et al., 1969; Data Project, 2008). The manners of analysis that were established were: occurrence, percentage, coefficient, ratio, and efficiency (Ejem, 1980; Palao, 2008; Schleuder, 1998).

Table 1. List of actions, criteria, and manners of analysis established after stage one

\begin{tabular}{|c|c|c|c|c|c|}
\hline Serve & Reception & Set & Spike & Block & Dig \\
\hline Occurrence & Occurrence & Occurrence & Occurrence & Occurrence & Occurrence \\
\hline Percentage & Percentage & Percentage & Percentage & Percentage & Percentage \\
\hline Coefficient & Coefficient & Coefficient & Coefficient & Coefficient & Coefficient \\
\hline $\begin{array}{c}\text { Point-to-error ratio } \\
\text { Efficiency }\end{array}$ & Efficiency & Efficiency & Efficiency & Efficiency & Efficiency \\
\hline Technique & Serve type & Technique & Technique & Technique & Technique \\
\hline Zone & Technique & Zone & Zone & Zone & Zone \\
\hline Tempo & Zone & Tempo & Tempo & Tempo & Tempo \\
\hline Complex & Tempo & Complex & Complex & Complex & Complex \\
\hline Player role & $\begin{array}{l}\text { Complex } \\
\text { Player role }\end{array}$ & $\begin{array}{c}\text { Player role } \\
\text { Recep/dig efficacy }\end{array}$ & $\begin{array}{c}\text { Player role } \\
\text { Recep/dig efficacy }\end{array}$ & $\begin{array}{c}\text { Player role } \\
\text { Recep/dig efficacy }\end{array}$ & Player role \\
\hline
\end{tabular}

In the second stage, after the data collection and data analysis, significant differences were found in the variables presented in Table 2. In the third stage, a comparison of the results obtained with data from previous studies in the literature (normal values and win/loss studies) was done (Table 3 ).

Table 2. Differences between the teams' won and lost sets in the previous season (data from match analysis of Spain's professional first division volleyball players)

\begin{tabular}{lccccc} 
& \multicolumn{2}{c}{ Won set } & \multicolumn{3}{c}{ Lost set } \\
\cline { 2 - 6 } & M & SD & M & SD & Sig. \\
\hline 1 & 2 & 3 & 4 & 5 & 6 \\
\hline Serve (Coefficient) & 1.44 & 0.19 & 1.36 & 0.25 & 0.126 \\
Serve (Efficiency) & 23.37 & 6.70 & 17.24 & 16.11 & $0.049^{*}$ \\
Serve (Error percentage) & 9.92 & 6.12 & 13.71 & 6.78 & $0.008^{*}$ \\
\hline
\end{tabular}




\begin{tabular}{|c|c|c|c|c|c|}
\hline 1 & 2 & 3 & 4 & 5 & 6 \\
\hline Reception (Coefficient) & 2.14 & 0.16 & 2.04 & 0.19 & $0.006^{*}$ \\
\hline Reception (Efficiency) & 21.16 & 14.69 & 17.30 & 16.00 & 0.249 \\
\hline Spike (Coefficient) & 2.87 & 0.35 & 2.60 & 0.38 & $0.001^{*}$ \\
\hline Spike (Efficiency) & 37.39 & 21.24 & 24.54 & 19.77 & $0.005^{*}$ \\
\hline Block (Coefficient) & 1.62 & 0.39 & 1.25 & 0.38 & $0.001^{*}$ \\
\hline Block (Efficiency) & 8.21 & 15.45 & 0.84 & 17.10 & $0.039^{*}$ \\
\hline Dig (Coefficient) & 1.09 & 0.34 & 0.90 & 0.26 & $0.006^{*}$ \\
\hline Dig (Efficiency) & -34.49 & 20.95 & -44.14 & 15.55 & 0.021 \\
\hline Counter-attack (Coefficient) & 2.66 & 0.44 & 2.42 & 0.56 & $0.040^{*}$ \\
\hline Counter-attack (Efficiency) & 26.12 & 23.77 & 13.59 & 27.49 & $0.026^{*}$ \\
\hline
\end{tabular}

Legend: * - Student t-test for independent samples. Coefficient: average efficacy score of an action on a scale from 0 to 4 for terminal actions and from 0 to 3 for actions of continuity). Efficiency - Percentage of Perfect/Point actions - Percentage of errors (for the serve, the actions that limit the opponent attack were also included; for the dig, the actions that allow the team attack were also included).

Table 3. Differences between winning and losing (data from the review of the literature)

\begin{tabular}{|c|c|c|c|}
\hline & Won set & Lost set & Sig. \\
\hline Serve (Coefficient) & $1.36^{\mathrm{a}-1.44^{\mathrm{b}}}$ & $1.15^{b}-1.27^{a}$ & *a \\
\hline Serve (Efficiency) & $-8.8^{\mathrm{b}}$ & $-11.0^{\mathrm{b}}$ & *a \\
\hline Serve (Error percentage) & $12.7^{\mathrm{b}}$ & $13.8^{\mathrm{b}}$ & *a \\
\hline Reception (Coefficient) & $2.40^{a}-2.59^{b}$ & $2.29^{a}-2.51^{b}$ & *a \\
\hline Reception (Efficiency) & $62.9^{b}$ & $56.4^{b}$ & $*_{a}$ \\
\hline Spike (Coefficient) & $2.64^{\mathrm{a}}-2.99^{\mathrm{b}}$ & $2.50^{b}-2.66^{a}$ & $\star_{a, b}$ \\
\hline Spike (Efficiency) & $31.3^{b}$ & $25.5^{\mathrm{b}}$ & $\star_{a, b}$, \\
\hline Block (Coefficient) & $1.29^{b}-1.84^{a}$ & $1.18^{b}-1.58^{a}$ & $*_{a, b}$ \\
\hline Block (Efficiency) & $-11.9^{b}$ & $-16.2^{b}$ & $\star_{\mathrm{a}, \mathrm{b}}$ \\
\hline Dig (Coefficient) & $1.85^{b}$ & $1.77^{\mathrm{b}}$ & \\
\hline Dig (Efficiency) & $-1.4^{b}$ & $0.3^{b}$ & \\
\hline Counter-attack (Efficiency) & $2.92^{\mathrm{a}}$ & $2.57^{\mathrm{a}}$ & *a \\
\hline Dig (Coefficient) & - & - & *a \\
\hline
\end{tabular}

Legend: Efficiency - Percentage of perfect/point actions minus percentage of errors actions (for the serve, the actions that limit the opponent attack were also included; for the dig, the actions that allow the team attack were also included).

Note: Only studies that analyzed differences between winning and losing or differences between level of competition by sets or complex phases were included ("Marelić, Rešetar,, Janković, 2004; ' Palao, Santos, Ureña, 2004).

After the review of the data done by the coaches and researchers, the list of reference values was established (Table 4). The expert panel did not eliminate any skills. The analysis by phase only was considered for two skills. Rotation criteria was not given consideration. Only three manners of analysis were finally selected (occurrence, percentage, and efficacy) to ease understanding for the players. The list was reduced to seven performance goals. Coaches' criteria were that in order for goals to be applicable to evaluate players in practice and in competition, there should be few. The serve was the only action with two analysed criteria. The spike was analysed in two-phase moments (side-out and counter-attack). A simulation of the performance goals using info from previous matches was used to test the performance goals during this stage. 
Table 4. Technical and tactical performance goals for male performance volleyball players (data from match analysis of Spain's professional first division men's volleyball players).

\begin{tabular}{lcc}
\hline \multicolumn{1}{c}{ Technique } & Criteria & Goal \\
\hline Reception & Efficacy (Perfect - Error) & $55 \%$ \\
Attack & Efficacy (Points - Error) & $40-45 \%$ \\
Counter-attack & Efficacy (Points - Error) & $20-25 \%$ \\
Serve & Percentage of error & $9 \%$ \\
Serve & Percentage of points and actions that reduce the opponent attack options & $35-40 \%$ \\
Block & Number of contacts per set & 3 points \& 3 contacts that allow team to continue playing \\
Dig & Number of contacts per set & 6 contacts that allow team to continue playing \\
\hline
\end{tabular}

\section{Discussion}

The goal of this study was to establish technical and tactical performance goals for elite male volleyball players (Spain's first division). In the first stage, the experts suggested many options for possible analysis. Ideas from research and previous practical experiences were discussed and reviewed to establish the data needed and the analysis necessary to obtain these data. Researchers' collaboration previous experience with coaches was useful (e.g. Palao, López-Martínez, 2012). To gather ideas and perspectives and promote discussion between the expert panel, several studies and books were reviewed related to the nature of volleyball, related to game complex or the order of actions and related to types of data analysis (e.g. Eom, Schutz, 1992ab; Ejem, 1980; Diaz, 1992; Palao, 2004, 2008; Palao, Manzanares, 2009; Schleuder, 1998). The criteria used to analyze the viability of brainstorm ideas were if they could be adapted to the statistical system used by coaches with their teams (software, human resources, possibility to integrate information into practices and competitions, etc.). It was difficult to unify criteria for the set action in stage one. Different coaches had different ways of understanding this action of the game.

From the first stage, a protocol to extract the data from the software used by the coaches to collect and analyze their teams (Data Volley) was developed. More information about this protocol can be found in Garcíade-Alcaraz and Palao (2013). This allowed us to extract the data from match analysis software and use statistical packets for the data analysis regarding the results of the set. From the data analysis, the importance of the sideout actions was found. The researchers reviewed the data from the second stage with the data from the research studies found in the bibliography. A report with all these data was presented by the researcher to the expert panels. The possibilities for comparing the data obtained in the second stage with the data from research studies were limited because not all the studies utilized the same unit of analysis (e.g. match, set or rally) or the same way to present the data (e.g. occurrence, percentage, etc.); further, some studies only presented the inferential values. This affects the possibilities coaches have for using the information from these studies by coaches.

The last stage attempted to establish practical outcomes of the work done in previous stages. In this stage, the coaches emphasized that in order to use these performance goals they must be realistic, adjust to their work system, and few in number. For coaches, at least one goal from each skill must be included to have a general perspective of the game and give every skill the same importance. However, coaches decided not to include any goal for the set because of the high level of efficacy, and they included two performance goals each for the serve and spike. Coaches emphasized the importance of the set in the game, but they thought that these data were not 
useful enough or that the way to measure this action was difficult to be introduced into their statistical analysis. More studies are needed to analyze the setter's actions (e.g. number of blockers in front of the spiker, use of the jump set, etc.) and the viability of including this measurement in coaches' statistical analysis of the game. During the process of selecting the performance goals, the criteria used to decide which of the performance goals would be included in the final proposed criteria was to determine whether it could be implemented easily in team protocols and whether it would allow coaches to provide information to their players and teams (i.e. usefulness).

The collaboration of coaches and researchers is a key aspect to use scientific knowledge in real situations. This paper describes the stages carried out to apply protocol used in research to develop a proposal of technical and tactical performance goals. The authors believe that this type of collaboration allows us to improve the type of analysis done, get more applicable and useful results, etc. Performance analysis researchers must collaborate with coaches and athletes if they want their studies to be used by elite coaches. Coaches must also collaborate with researchers when they want to innovate, introduce new perspectives and analyses, and update their knowledge with evidence-based information. This type of collaboration contributes toward reducing the gap between sport professionals and researchers.

\section{Conclusions}

Seven performance goals were established to evaluate the technical-tactical actions of the players in training and in competition for Spain's first division men's volleyball teams or a similar level. Protocols utilized and data found can be used as references and as an example of how to calculate technical and tactical performance goals in sports. This paper shows a collaboration between researchers and coaches as an element that generates new applicable knowledge using scientific methods for the professional that uses the knowledge provided by scientific studies in performance analysis. The collaboration between researchers and coaches allowed for the integration of a conceptual analysis of the performance indicators (review of literature and expert analysis), a mathematical analysis of the performance indicators, and an application analysis (usefulness and integration in training). The combination of these approaches provided an applicable realistic outcome that is evidence-based. More studies and collaborations need to be done by researchers and coaches so their work can benefit from each other.

\section{Acknowledgment}

This work was supported by the Seneca Foundation (Región de Murcia, Spain) under Grant 08632/PPC/08.

\section{References}

Bull, S.J., Albinson, J.G., Shambrook, C.J. (1996). The mental game plan. Eastbourne: Sports Dynamics.

Coleman, J. (2002). Scouting opponents and evaluating team performance. In: D.S. Shondell, C. Reynaud (eds.), The volleyball coaching bible (pp. 321-346). Champaign, IL: Human Kinetics.

Coleman, J.E., Neville, B., Gordon, B. (1969). A statistical system for volleyball and its use in Chicago Women's Association. International Volleyball Review, 17, 72-73.

Data Project (2008). Handbook of Data Volley. Bolgnia: Data project.

Díaz, J. (1992). Voleibol. La dirección de equipo, $2^{a}$ Edición. Sevilla: Wanceulen.

Ejem, M. (1980). Some theoretical aspects of statistical game analysis utilization in player's performance evaluation. Volleyball Technical Journal, 3 (5), 43-48. 
Eom, H.J., Schutz, R.W. (1992a). Statistical analyses of volleyball team performance. Research Quarterly for Exercise and Sport, 1 (63), 11-18.

Eom, H.J., Schutz, R.W. (1992b). Transition play in team performance of volleyball: A log-linear analysis. Research Quarterly for Exercise and Sport, 3 (63), 261-269.

García de Alcaraz, A. Palao, J.M. (2013). Protocolo para la realización y exportación de análisis específicos dentro del software de análisis de juego en voleibol 'Data Volley'. Lecturas: Educación Física y Deportes (http://www. efdeportes.com), 176.

Marelić, N., Rešetar, T., Janković, V. (2004). Discriminant analysis of the sets won and the sets lost by one team in A1 Italian volleyball league-A case study. Kineziologija, 1 (36), 75-82.

Palao, J.M. Manzanares, P. (2009). Manual del instrumento de observación de las técnicas y la eficacia en voleibol (TEVOL - VERSIÓN 1.0). Murcia, Spain: Self-published.

Palao, J.M. (2004). Incidencia de los complejos de juego y la posición del colocador sobre el rendimiento en competición. Rendimiento deportivo, 9, 1-19.

Palao, J.M. (2008). Options for analysis of the volleyball score sheet. International Journal of Performance Analysis in Sport, 2 (8), 26-43.

Palao, J.M., López-Martínez, A.B. (2012). Establecimiento de objetivos a partir del análisis del juego para el trabajo técnico-táctico en voley-playa. Un caso práctico. Revista de Eduación Física, 396, 35-47.

Palao, J.M., Santos, J.A., Ureña, A. (2004). Effect of team level on skill performance in volleyball. International Journal of Performance Analysis of Sport, 2 (4), 50-60.

Samozino, P., Rejc, E., Di Prampero, P.E., Belli, A., Morin, J.B. (2012). Optimal force-velocity profile in ballistic movements-altius: citius or fortius? Medicine \& Science in Sports \& Exercise, 2 (44), 313-322.

Schleuder, S. (1998). Comprehensive volleyball statistics: a guide for coaches, media and fans. Colorado Springs, CO: Volleyball Informational Products.

Williams, S.J., Kendall, L. (2007). Perceptions of elite coaches and sports scientists of the research needs for elite coaching practice. Journal of Sports Sciences, 14 (25), 1577-1586.

Cite this article aS: Palao, J.M., Garcia-de-Alcaraz, A., Hernández-Hernández, E., Valadés, D., Ortega-Toro, E. (2018). Establishing Technical and Tactical Performance Goals for Elite Male Volleyball Players. Central European Journal of Sport Sciences and Medicine, 1 (21), 5-12. DOI: 10.18276/cej.2018.1-01. 